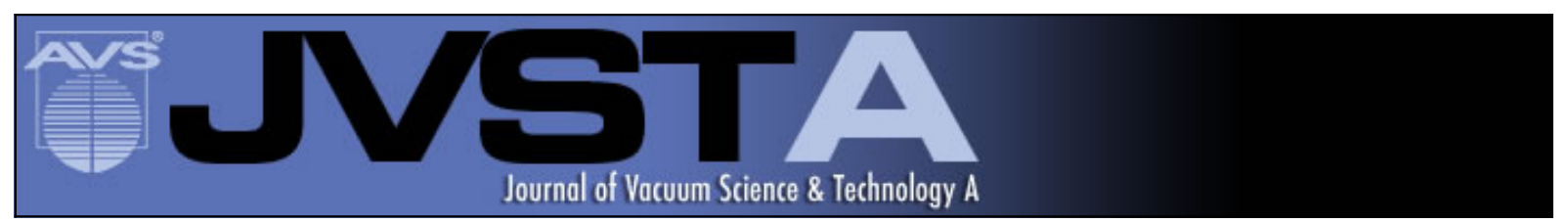

\title{
Planar regions of GaAs (001) prepared by Ga droplet motion
}

Changxi Zheng, Wen-Xin Tang, and David E. Jesson

Citation: Journal of Vacuum Science \& Technology A 34, 043201 (2016); doi: 10.1116/1.4948530

View online: http://dx.doi.org/10.1116/1.4948530

View Table of Contents: http://scitation.aip.org/content/avs/journal/jvsta/34/4?ver=pdfcov

Published by the AVS: Science \& Technology of Materials, Interfaces, and Processing

\section{Articles you may be interested in}

Kinetic Monte Carlo simulation of GaAs(001) MBE growth considering the V/III flux ratio effect

J. Vac. Sci. Technol. B 34, 041804 (2016); 10.1116/1.4948514

Planar InAs growth on $\mathrm{GaAs}(001)$ and subsequent quantum dot formation by a surface induced morphological instability

J. Vac. Sci. Technol. B 22, 2261 (2004); 10.1116/1.1771671

Structure and morphology of the As-rich and the stoichiometric GaAs(114)A surface

J. Appl. Phys. 95, 7645 (2004); 10.1063/1.1707212

Molecular-beam epitaxy of $\mathrm{Ge}$ on $\mathrm{GaAs}(001)$ and $\mathrm{Si}$ capping

J. Appl. Phys. 93, 3057 (2003); 10.1063/1.1542656

Nitridation of the GaAs (001) surface using atomic nitrogen

J. Vac. Sci. Technol. B 15, 1133 (1997); 10.1116/1.589427

\section{HDDEN}

\section{Instruments for Advanced Science}

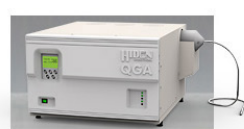

Gas Analysis

dynamic measurement of reaction gas streams catalysis and thermal analysis

'molecular beam studies

, dssolved species probes

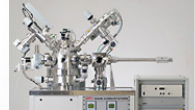

Surface Science

, UHVTPD

SIMS elemental imaging - surface mapping

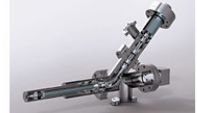

Plasma Diagnostics plasma source characterization etch and deposition process reaction analysis of neutral and radical species

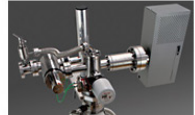

Vacuum Analysis ' partial pressure measurement and control of process gases reactive sputter process contro , vacuum diagnostics , vacuum coating process monitoring 


\title{
SHOP NOTES
}

These are "how to do it" papers. They should be written and illustrated so that the reader may easily follow whatever

instruction or advice is being given.

\section{Planar regions of GaAs (001) prepared by Ga droplet motion}

\author{
Changxi Zheng ${ }^{\text {a) }}$ \\ Department of Civil Engineering, Monash University, Victoria 3800, Australia \\ Wen-Xin Tang \\ College of Materials Science and Engineering, Chongqing University, Chongqing 400044, \\ People's Republic of China and School of Physics and Astronomy, Monash University, \\ Victoria 3800, Australia \\ David E. Jesson ${ }^{\text {b) }}$ \\ School of Physics and Astronomy, Cardiff University, Cardiff CF24 3AA, United Kingdom
}

(Received 4 November 2015; accepted 21 April 2016; published 10 May 2016)

\begin{abstract}
The authors describe a simple method for obtaining planar regions of GaAs (001) suitable for surface science studies. The technique, which requires no buffer layer growth, atomic hydrogen source, or the introduction of As flux, employs controllable Ga droplet motion to create planar trail regions during Langmuir evaporation. Low-energy electron microscopy/diffraction techniques are applied to monitor the droplet motion and characterize the morphology and the surface reconstruction. It is found that the planar regions exhibit atomic flatness at the level of a high-quality buffer layer. (C) 2016 Author(s). All article content, except where otherwise noted, is licensed under a Creative Commons Attribution (CC BY) license (http://creativecommons.org/ licenses/by/4.0/). [http://dx.doi.org/10.1116/1.4948530]
\end{abstract}

\section{INTRODUCTION}

GaAs surfaces are of appreciable scientific and technological importance and have been actively studied over the years. ${ }^{1-3}$ The preparation of clean, atomically flat surfaces is paramount for fundamental studies of surface energetics, quantum structure formation, and epitaxial growth. ${ }^{1-3}$ With the emergence of in situ characterization techniques, such as transmission ${ }^{4}$ and low energy electron microscopy (LEEM),${ }^{5,6}$ it is desirable to establish a simple means of creating planar surfaces for fundamental studies which do not demand a high As overpressure and which are compatible with the deposition geometry limitations imposed by electron optics. Here, we describe a simple method of smoothing GaAs (001) which utilizes the motion of $\mathrm{Ga}$ droplets and does not require material deposition.

The conventional approach to preparing planar GaAs (001) surfaces involves high temperature $\left(\sim 600^{\circ} \mathrm{C}\right)$ thermal desorption of the native oxide followed by the growth of thick $(>0.5 \mu \mathrm{m}) \mathrm{GaAs}$ buffer layers and surface annealing under As flux. ${ }^{1-3}$ This approach cannot be utilized if buffer layer growth is not possible or if the introduction of As flux is undesirable. The alternative approach of atomic hydrogen $(\mathrm{AH})$ cleaning $^{7}$ offers a number of advantages, but annealing under As flux followed by subsequent buffer layer growth is ideally required to optimize the surfaces. ${ }^{8}$ Depending on the

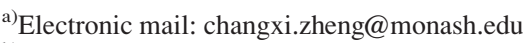

b)Electronic mail: jessonDE@cardiff.ac.uk
}

system design and geometry, it may not be possible or convenient to introduce such an $\mathrm{AH}$ source or indeed such equipment might not be available. Our method to create planar regions using $\mathrm{Ga}$ droplet motion involves no deposition or buffer layer growth and is compatible with all systems equipped with a heating stage.

\section{EXPERIMENT}

Experiments were performed in an ultrahigh-vacuum (UHV) LEEM. $^{9}$ Temperatures $T$ were measured using an infrared pyrometer, which incorporated a correction due to the $T$-dependence of the surface emissivity ${ }^{10}$ and was calibrated to the congruent evaporation temperature of $\mathrm{GaAs}$ given by $T_{c}=625^{\circ} \mathrm{C} .{ }^{11,12} \mathrm{We}$ degassed a (001)-oriented undoped $\mathrm{GaAs}$ sample at $300^{\circ} \mathrm{C}$ for $24 \mathrm{~h}$. This was followed by high temperature flashing up to $600{ }^{\circ} \mathrm{C}$ and annealing at $580^{\circ} \mathrm{C}$ for $2 \mathrm{~h}$ to remove the surface oxide. This procedure necessarily creates a rough surface [Fig. 1(a)].

Our method to prepare droplets is based on the thermodynamics of Ga droplets on GaAs (001). During congruent evaporation $\left(T<T_{c}\right)$, Ga and As evaporate at equal rates from the surface and the Ga surface chemical potential $\mu_{\mathrm{Ga}}$ attains a steady-state value. With increasing temperature, $\mu_{\mathrm{Ga}}$ will increase to make As and Ga evaporation rates equal but eventually reaches the Ga liquidus value $\mu_{L}$ which defines the upper limit $T=T_{c}$ for congruent evaporation. Above $T_{c}, \mu_{\mathrm{Ga}}>\mu_{L}$ so that excess Ga can collect as droplets which are assumed to remain close to liquidus composition, 

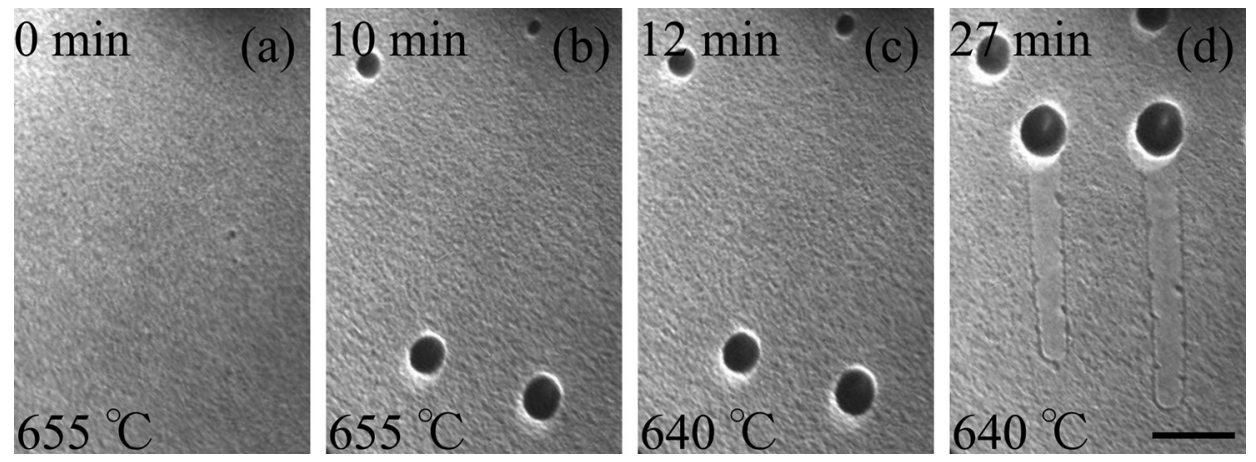

FIG. 1. MEM images of Ga droplet formation and dynamics. (a) Rough GaAs (001) surface following removal of the oxide by thermal annealing. (b) The surface is then annealed at $655^{\circ} \mathrm{C}$ for 10 min to generate Ga droplets. (c) The surface is rapidly cooled to $640{ }^{\circ} \mathrm{C}$ (within 2 min) and annealed at this temperature for $15 \mathrm{~min}$ (d) so that the droplets grow and move across the surface to create trails. The scale marker is $5 \mu \mathrm{m}$ across.

in equilibrium with the GaAs substrate. The droplets therefore act as sinks for surface $\mathrm{Ga}$ adatoms which pins $\mu_{\mathrm{Ga}}$ close to $\mu_{L}$. This prevents the increase in $\mu_{\mathrm{Ga}}$ so that above $T_{c}$, As evaporates more rapidly than $\mathrm{Ga}$ and the Ga droplets grow.

\section{RESULTS AND DISCUSSION}

Annealing the surface shown in Fig. 1(a) at $655^{\circ} \mathrm{C}$ for $10 \mathrm{~min}$ creates four droplets in the field of view [Fig. 1(b)]. In mirror electron microscopy (MEM), the droplets appear as dark disks, somewhat larger than the actual droplet size, enclosed by a bright ring. ${ }^{13}$ Further droplet nucleation is prevented by rapidly reducing the substrate temperature to $640^{\circ} \mathrm{C}$ [Fig. 1(c)]. Annealing at this temperature causes the droplets to grow, but their number remains constant. By adjusting the temperature in this way, it is possible to control the droplet density and size to optimize the smoothing procedure.
The droplets in Fig. 1(c), at liquidus composition, are out of equilibrium with the surrounding surface and this produces a force on the droplet proportional to its diameter. ${ }^{14}$ When the droplets attain a large enough size, this force overcomes the pinning force due to droplet etching ${ }^{15}$ and the droplets move along \pm [110] directions, leaving behind smooth trails [Fig. 1(d)]. The droplet size controls the trail width, and the anneal time controls its length. Annealing the sample for $15 \mathrm{~min}$ produces trail lengths of up to tens of microns [Fig. 1(d)].

Figure 2(a) shows an LEEM image of the trail which consists of planar terrace regions separated by monolayer surface steps. The step density is smaller than $15 \mu \mathrm{m}^{-1}$, and the surface morphology is comparable to that obtained by thick buffer layer growth. ${ }^{1}$ The micro-low-energy electron diffraction ( $\mu$ LEED) pattern from the trail contained in Fig. 2(b) corresponds to a $c(8 \times 2)$ reconstruction. This is in contrast to the $\mu$ LEED pattern of the region outside the trail which, in addition
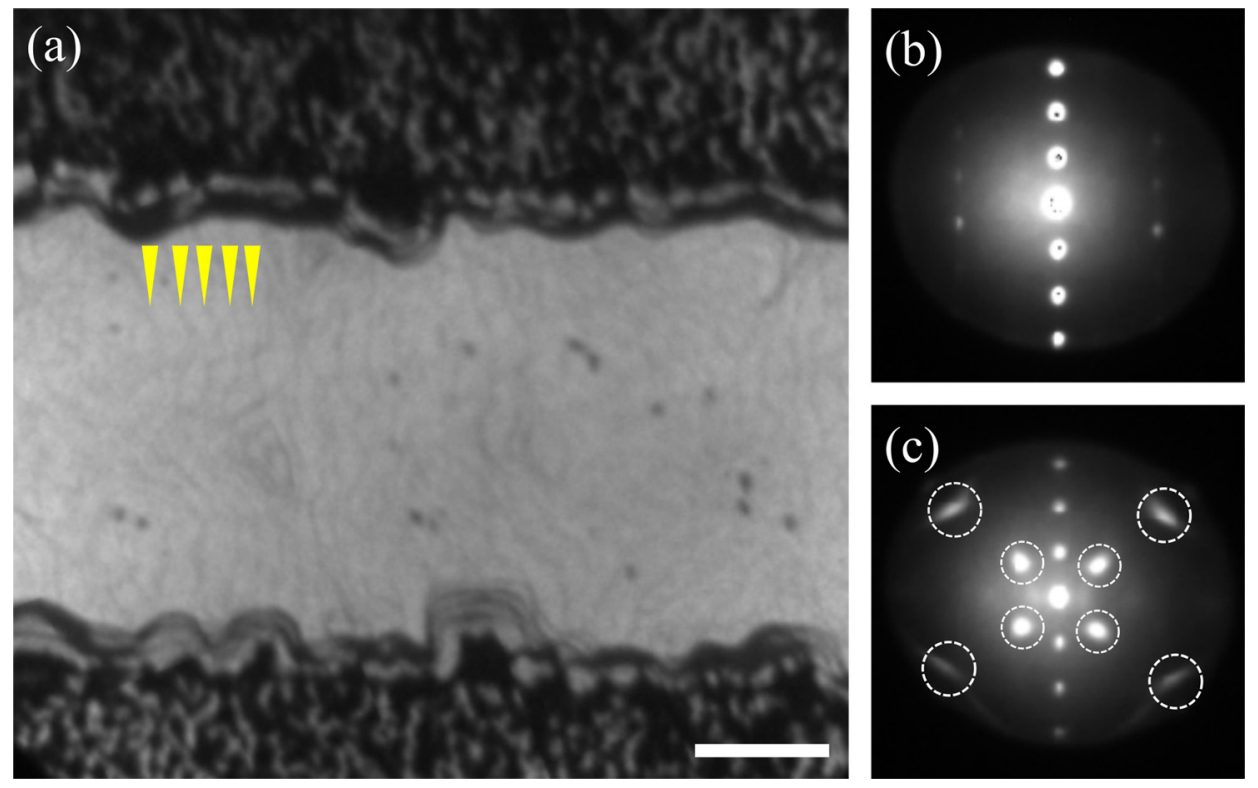

FIG. 2. (Color online) (a) LEEM image of a GaAs (001) surface at $560^{\circ} \mathrm{C}$ where a trail has been created by a running droplet. The arrows indicate monolayer height surface steps. (b) Corresponding $\mu$ LEED pattern obtained inside the trail. The well-defined spots indicate a $\mathrm{c}(8 \times 2)$ surface reconstruction. (c) Corresponding $\mu$ LEED pattern obtained from the rough surface outside of the trail. In addition to $c(8 \times 2)$ spots, extra facet spots (circled) are present which change position with electron beam energy. The electron energy for LEEM imaging was $2.4 \mathrm{eV}$ and $10.3 \mathrm{eV}$ for LEED diffraction. The scale bar is $1 \mu \mathrm{m}$. 
to $\mathrm{c}(2 \times 8)$ spots, also contains additional spots which move with electron beam energy and can be linked to facets present in the surface roughness. ${ }^{16}$ The general roughness and associated facets are related to thermal oxide desorption followed by long-time surface annealing at high temperature. It can be seen that running droplets are effective tools for removing this roughness and creating atomically flat, planar regions for surface science studies. Although it is known that buffer layers can additionally bury low-level chemical impurities, it is presently unclear as to whether running droplets can provide similar effects by surface absorption.

By a judicious choice of sample heating to control droplet nucleation and motion, it is possible to approach a smooth surface coverage of $90 \%$. Droplets can then be removed by annealing below $T_{c}$. Even higher percentages of smooth surface can be obtained by droplet nucleation and motion, but this generally involves the formation of large $(\geq 10 \mu \mathrm{m})$ droplets which are impractical to remove by annealing below $T_{c}$ due to the protracted times involved. Nevertheless, such large areas of smooth surface open up the possibility of using chemical spectroscopies, or standard surface electron diffraction techniques. Smaller trail regions, as in Fig. 2, for example, are suitable for the study by local and semilocal probes such as the scanning tunneling microscopy, scanning electron microscopy, and LEEM. Moreover, droplet motion and control has been observed across a variety of III-V material systems, ${ }^{17-21}$ which may lead to broader applications of the method discussed here.

\section{SUMMARY AND CONCLUSIONS}

In summary, we have described a simple method for obtaining planar regions of GaAs (001) suitable for surface science studies. The method requires no buffer layer growth, AH source, or the introduction of As flux. If real space imaging is available, the process can be fine-tuned by controlling the temperature for droplet formation and motion. However, ex situ analysis of test surfaces annealed at different temperatures should allow this method to be routinely used in any UHV system.

\section{ACKNOWLEDGMENTS}

C.Z. acknowledges support from Australian Research Council (ARC) and D.E.J. acknowledges support from a Marie Curie International Incoming Fellowship.

${ }^{1}$ A. Ohtake, Surf. Sci. Rep. 63, 295 (2008).

${ }^{2}$ V. P. LaBella, M. R. Krause, Z. Ding, and P. M. Thibado, Surf. Sci. Rep. 60, 1 (2005).

${ }^{3}$ B. A. Joyce and D. D. Vvedensky, Mater. Sci. Eng., R 46, 127 (2004).

${ }^{4}$ R. M. Tromp and F. M. Ross, Annu. Rev. Mater. Sci. 30, 431 (2000).

${ }^{5}$ E. Bauer, Rep. Prog. Phys. 57, 895 (1994).

${ }^{6}$ M. S. Altman, J. Phys. Condens. Matter 22, 084017 (2010).

${ }^{7}$ K. G. Tschersich and V. von Bonin, J. Appl. Phys. 84, 4065 (1998).

${ }^{8}$ A. Khatiri, T. J. Krzyzewski, C. F. McConville, and T. S. Jones, J. Cryst. Growth 282, 1 (2005).

${ }^{9}$ D. E. Jesson and W. X. Tang, Microscopy: Science, Technology, Applications and Education, edited by A. Mendez-Vilas and J. Diaz (Formatex Research Center, Badajoz, 2010), Vol. 3, p. 1608.

${ }^{10}$ P. J. Timans, J. Appl. Phys. 72, 660 (1992).

${ }^{11} \mathrm{~J}$. Y. Tsao, Materials Fundamentals of Molecular Beam Epitaxy (Academic, San Diego, CA, 1993).

${ }^{12}$ Z. Y. Zhou, C. X. Zheng, W. X. Tang, D. E. Jesson, and J. Tersoff, Appl. Phys. Lett. 97, 121912 (2010).

${ }^{13}$ S. M. Kennedy, C. X. Zheng, W. X. Tang, D. M. Paganin, and D. E. Jesson, Ultramicroscopy 111, 356 (2011).

${ }^{14}$ J. Tersoff, D. E. Jesson, and W. X. Tang, Science 324, 236 (2009).

${ }^{15}$ C. X. Zheng, W. X. Tang, and D. E. Jesson, Appl. Phys. Lett. 100, 071903 (2012).

${ }^{16}$ T. Yasue, T. Koshikawa, M. Jalochowski, and E. Bauer, Surf. Sci. 493, 381 (2001).

${ }^{17}$ E. Hilner, A. A. Zakharov, K. Schulte, P. Kratzer, J. N. Andersen, E. Lundgren, and A. Mikkelsen, Nano Lett. 9, 2710 (2009).

${ }^{18}$ J. Wu, Z. M. Wang, A. Z. Li, M. Benamara, S. Li, and G. J. Salamo, PLoS One 6, e20765 (2011).

${ }^{19}$ S. Kanjanachuchai and P. Photongkam, Cryst. Growth Des. 15, 14 (2015).

${ }^{20}$ A. A. Zakharov, E. Mårsell, E. Hilner, R. Timm, J. N. Andersen, E. Lundgren, and A. Mikkelsen, ACS Nano 9, 5422 (2015).

${ }^{21}$ B. A. Trisna, N. Nakareseisoon, W. Eiwwongcharoen, S. Panyakeow, and S. Kanjanachuchai, Nanoscale Res. Lett. 10, 184 (2015). 